\title{
Whole-transcriptome analysis of differentially expressed genes in the mutant and normal capitula of Chrysanthemum morifolium
}

Hua Liu', Chang Luo ${ }^{1}$, Dongliang Chen ${ }^{1}$, Yaqin Wang ${ }^{2}$, Shuang Guo ${ }^{1}$, Xiaoxi Chen ${ }^{1}$, Jingyi Bai ${ }^{1}$, Mingyuan Li ${ }^{1}$, Xinlei Huang ${ }^{1}$, Xi Cheng ${ }^{1}$ and Conglin Huang ${ }^{1 *}$ (D)

\begin{abstract}
Background: Chrysanthemum morifolium is one of the most economically important and popular floricultural crops in the family Asteraceae. Chrysanthemum flowers vary considerably in terms of colors and shapes. However, the molecular mechanism controlling the development of chrysanthemum floral colors and shapes remains an enigma. We analyzed a cut-flower chrysanthemum variety that produces normal capitula composed of ray florets with normally developed pistils and purple corollas and mutant capitula comprising ray florets with green corollas and vegetative buds instead of pistils.

Results: We conducted a whole-transcriptome analysis of the differentially expressed genes (DEGs) in the mutant and normal capitula using third-generation and second-generation sequencing techniques. We identified the DEGs between the mutant and normal capitula to reveal important regulators underlying the differential development. Many transcription factors and genes related to the photoperiod and GA pathways, floral organ identity, and the anthocyanin biosynthesis pathway were differentially expressed between the normal and mutant capitula. A qualitative analysis of the pigments in the florets of normal and mutant capitula indicated anthocyanins were synthesized and accumulated in the florets of normal capitula, but not in the florets of mutant capitula. These results provide clues regarding the molecular basis of the replacement of Chrysanthemum morifolium ray florets with normally developed pistils and purple corollas with mutant ray florets with green corollas and vegetative buds. Additionally, the study findings will help to elucidate the molecular mechanisms underlying floral organ development and contribute to the development of techniques for studying the regulation of flower shape and color, which may enhance chrysanthemum breeding.

(Continued on next page)
\end{abstract}

\footnotetext{
* Correspondence: conglinh@126.com

${ }^{1}$ Beijing Agro-Biotechnology Research Center, Beijing Academy of Agriculture and Forestry Sciences, Beijing Engineering Research Center of Functional Floriculture, Beijing, Key Laboratory of Agricultural Genetic Resources and Biotechnology, Beijing 100097, China

Full list of author information is available at the end of the article
}

C C The Author(s). 2021 Open Access This article is licensed under a Creative Commons Attribution 4.0 International License, which permits use, sharing, adaptation, distribution and reproduction in any medium or format, as long as you give appropriate credit to the original author(s) and the source, provide a link to the Creative Commons licence, and indicate if changes were made. The images or other third party material in this article are included in the article's Creative Commons licence, unless indicated otherwise in a credit line to the material. If material is not included in the article's Creative Commons licence and your intended use is not permitted by statutory regulation or exceeds the permitted use, you will need to obtain permission directly from the copyright holder. To view a copy of this licence, visit http://creativecommons.org/licenses/by/4.0/. The Creative Commons Public Domain Dedication waiver (http://creativecommons.org/publicdomain/zero/1.0/) applies to the data made available in this article, unless otherwise stated in a credit line to the data. 
(Continued from previous page)

Conclusions: The whole-transcriptome analysis of DEGs in mutant and normal C. morifolium capitula described herein indicates the anthocyanin deficiency of the mutant capitula may be related to the mutation that replaces ray floret pistils with vegetative buds. Moreover, pistils may be required for the anthocyanin biosynthesis in the corollas of chrysanthemum ray florets.

Keywords: Chrysanthemum morifolium, Ray florets, Pistils, Flower development, Mutant capitula, Anthocyanin biosynthesis, Whole-transcriptome analysis, Differentially expressed genes

\section{Background}

Chrysanthemum morifolium is one of the most economically important and popular floricultural crops in the family Asteraceae, and ranks second in the cut-flower industry after rose [1]. The head-like inflorescence (capitulum), which resembles a single large flower, is the main ornamental part of $C$. morifolium and is considered to be important for the evolutionary success of Asteraceae species [2]. The typical chrysanthemum capitulum is formed by two morphologically distinct florets, the marginal ray florets and the central disk florets. Ray florets have ligulate and zygomorphic colorful corollas (petals) and aborted stamens, which attract pollinators. The disk florets have radially symmetrical colorless corollas, and their fertile pollen grains are hermaphroditic and used for reproduction (Additional file 1). The diverse flower colors and shapes are the most visible results of floral evolution and have influenced the desirability of certain flowers to humans [3].

Flowering, which is a key developmental process in the plant life cycle, is a very complex process controlled by endogenous factors and environmental cues. More specifically, floral development comprises the following three phases: flowering determination, flower evocation, and floral organ development [4]. Regarding Arabidopsis thaliana, there has been substantial progress toward elucidating the molecular mechanisms underlying floral development $[5,6]$. The ABCDE models have revealed that A-class and E-class genes specify sepal identity; A-class, B-class, and E-class genes specify petal identity; B-class, $\mathrm{C}$-class, and E-class genes determine stamen identity; $\mathrm{C}$ class and E-class genes determine carpel/gynoecium organ identity; C-, E- and D-class genes specify ovule identity and differentiation [7]. With the notable exception of A-class genes, all of these genes belong to the MADS-box family of transcription factors, including the $A P 1, A P 3, P I, A G$, and SEP genes.

The diversity in plant colors, especially among flower petals, has enabled plants to continually develop new showy traits and prosper throughout millions of years of evolution. Anthocyanins and carotenoids are the two major groups of pigments generated in plant petals. Anthocyanins accumulate in the vacuoles of petal epidermal cells and confer orange-to-violet colors in flowers
[8]. In addition to attracting pollinators, anthocyanins also protect plants from UV irradiation [9]. Anthocyanins provide chrysanthemum ray florets with bright colors to attract pollinators, thereby increasing the cross pollination rate of different species or varieties and promoting the development of cultivar groups with highly variable flower types. Anthocyanins enhance the ornamental value of chrysanthemums, and many cut-flower and pot-flower varieties with bright colors are produced annually to satisfy market demands. Clarifying the mechanism regulating anthocyanin biosynthesis may enable researchers and breeders to produce novel chrysanthemum varieties with new flower colors.

In chrysanthemums, a few floral development regulatory genes have been isolated such as MADS-box, TCP, and WUS-like genes [10-13]. Some important functional genes and transcription factors involved in the anthocyanin biosynthesis pathway have also been characterized, including ANS, F3' $H, F 3 H$, and $M Y B$-like genes [13-16]. However, chrysanthemum capitula contain two morphologically distinct florets. Moreover, long-term breeding efforts have resulted in diverse flower shapes and colors. The mechanism underlying the evolution and development of chrysanthemum flowers is complex and remains relatively uncharacterized.

The development of RNA sequencing (RNA-seq) technology has greatly improved transcriptomic analyses of chrysanthemums [1]. However, the reads of secondgeneration high-throughput sequencing platforms are much shorter than the typical length of a eukaryotic mRNA. Additionally, the differences in transcript abundance and the presence of different unigenes make the assembly of transcriptomes from short reads extremely challenging [17]. Despite these problems, Hirakawa et al. used the Illumina sequencing platform for the de novo assembly of the whole Chrysanthemum seticuspe genome and Chi Song et al. sequenced the diploid Chrysanthemum nankingense genome using the Oxford Nanopore long-read technology [18, 19]. Unfortunately, no more than $40 \%$ of the transcriptome sequencing reads from $C$. morifolium can be mapped to these two genome sequences, probably because of the extreme variation in chromosome ploidy and biological characteristics. Third-generation sequencing technology has 
dramatically increased the length of sequencing reads, enabling the precise localization and sequencing of repetitive regions and unigenes with a single read.

We recently obtained a mutant plant of the cut-flower chrysanthemum variety C. morifolium ' $\mathrm{ZY}$ ' with both normal and mutant capitula. The normal capitula were composed of many rounds of ray florets with purple corollas and normally developed pistils, whereas in the mutant capitula, the ray floret corollas were green and the pistils were replaced by vegetative buds. In this study, we applied the Pacific Biosciences (PacBio) single-molecule long-read sequencing technology to analyze a mixed sample of normal and mutant flowers, leaves, stems, and roots from ' $\mathrm{ZY}$ ' plants. On the basis of the results, transcripts were sequenced and the mutant and normal capitula were examined using second-generation sequencing and RNA-seq technology. Thus, we combined third-generation and second-generation sequencing techniques to generate a more complete $C$. morifolium transcriptome.

Transcriptome sequencing and analysis revealed differentially expressed genes (DEGs) between the mutant and normal capitula, some of which may encode important regulators controlling the differential development. Many transcription factors and genes related to the photoperiod and gibberellin (GA) pathways, floral organ identity, and the anthocyanin biosynthesis pathway were differentially expressed between the normal and mutant capitula. These results may be useful for clarifying the molecular mechanisms underlying the phenotypic differences between ray florets with normally developed pistils and purple corollas and mutant ray florets with green corollas and vegetative buds in C. morifolium. Moreover, the data presented herein may elucidate the molecular basis of floral organ development, with implications for the development of techniques suitable for studying the regulation of flower shape and color and the breeding and molecular characterization of chrysanthemum.

\section{Results}

\section{Sequencing and assembly}

The C. morifolium 'ZY' plants analyzed in this study produced both normal and mutant capitula (Fig. 1). The normal capitula were composed of many rounds of ray florets with purple corollas and normally developed pistils. In contrast, the mutant capitula consisted of many rounds of mutant ray florets with green corollas as well as vegetative buds instead of pistils. We analyzed C. morifolium ' $\mathrm{ZY}$ ' normal and mutant capitula, leaves, stems, and roots using PacBio sequencing, after which the normal and mutant capitula were separately analyzed using Illumina paired-end sequencing technology. The resulting sequences were assembled into 130,097 unigenes

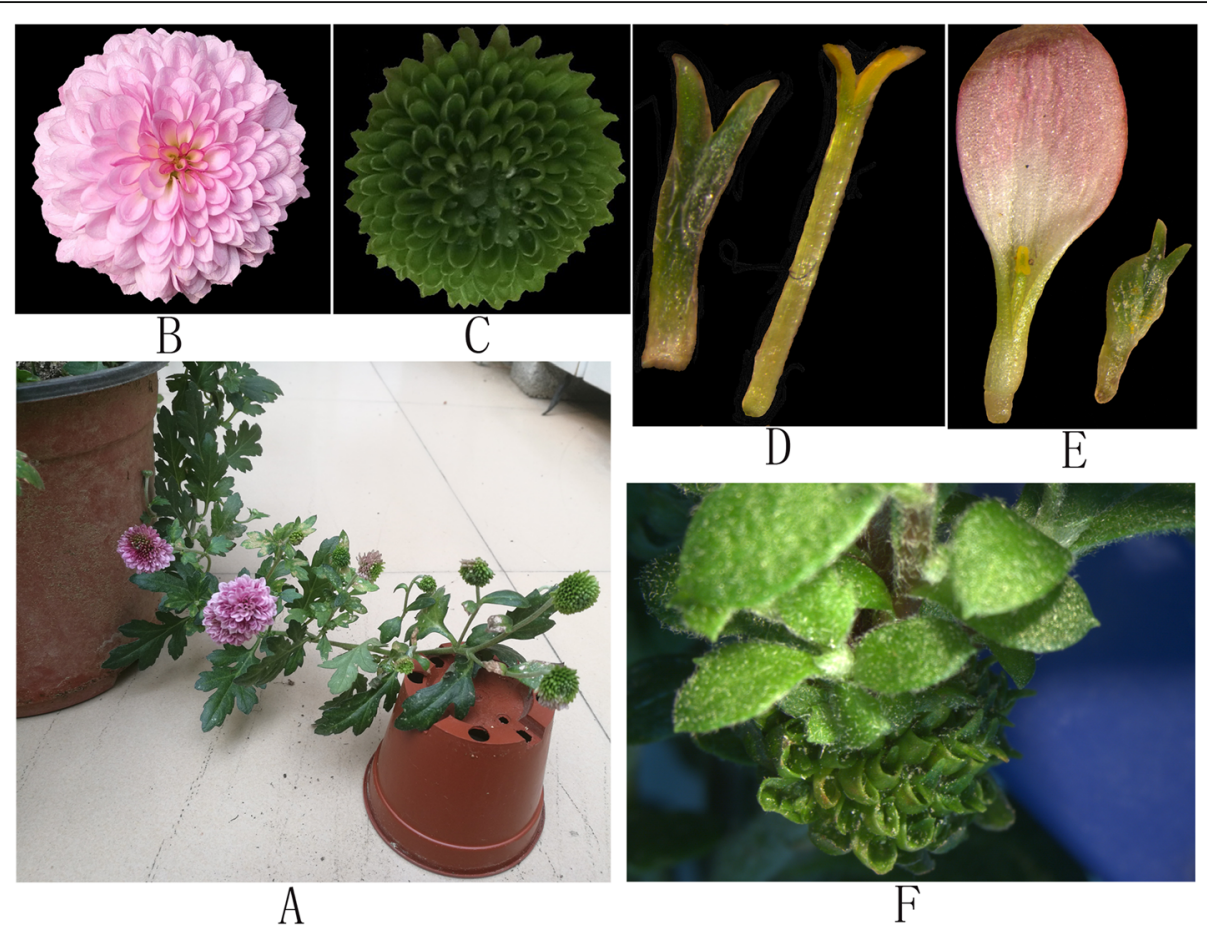

Fig. 1 Mutant Chrysanthemum morifolium ' $Z Y$ ' plant. (a) Mutant and normal capitula. (b) Normal capitulum. (c) Mutant capitulum. (d) Vegetative buds in the mutant capitulum (left) and pistils in the normal capitulum (right). (e) Normal ray florets (left) and mutant ray florets (right). (F) New shoots from the mutant capitulum 
with an N50 of $3013 \mathrm{bp}$ and an average length of 2510 bp (Table 1).

\section{Gene annotation and functional classification}

A total of 118,589 unigenes were annotated following a BLAST search of four databases [non-redundant (nr) protein database, Swiss-Prot, EuKaryotic Orthologous Groups (KOG), and Kyoto Encyclopedia of Genes and Genomes (KEGG)], leaving 11,508 (8.85\%) unannotated unigenes. A total of $118,043,101,048,87,630$, and 54,245 unigenes were annotated on the basis of searches of the nr, Swiss-Prot, KOG, and KEGG databases, respectively. Moreover, the Gene Ontology (GO) database was used for the functional annotation and analysis of genes, which were divided into the following three main categories: molecular function, cellular component, and biological process. Specifically, 36,144 unigenes were classified into 47 functional categories, including 19, 17, and 11 in the biological process, cellular component, and molecular function categories, respectively. The predominant biological process, molecular function, and cellular component GO terms among the genes were 'metabolic process' $(20,871)$, 'catalytic activity' $(22,818)$, and 'cell' $(11,887)$, respectively. This implied that numerous metabolic activities were activated during the development of chrysanthemum capitula in a process regulated by the combined effects of the proteins encoded by these diverse genes. Additionally, a substantial proportion of the genes were annotated with the 'cellular process', 'binding', and 'cell part' GO terms, whereas 'locomotion', 'transcription factor activity, protein binding', and 'extracellular matrix component' were relatively uncommon GO terms (Fig. 2).

The KOG database is usually used to identify orthologous and paralogous proteins. Additionally, JGIpredicted genes may be identified according to KOG classifications or IDs. The annotated sequences were used as queries to screen the KOG database to assess the completeness of our transcriptome library and the reliability of our annotation process. Of 118,043 nr hits, 87,630 sequences were assigned KOG classifications. Among the $25 \mathrm{KOG}$ categories, the cluster for 'general function prediction only' $(28,904,32.98 \%)$ represented the largest group, followed by 'signal transduction mechanisms' $(23,030,26.68 \%)$ and 'posttranslational modification, protein turnover, chaperones' $(19,436,22.18 \%)$. Conversely, the 'defense mechanisms' (673, 0.77\%), 'extracellular structures' (454,0.52\%), and 'cell motility' $(170,0.19 \%)$ clusters were the smallest groups (Fig. 3).
To further evaluate the chrysanthemum transcriptome, all unigenes were aligned with the sequences in the KEGG database using the BLASTx algorithm (E-value < $10^{-5}$ ). As a collection of manually drawn pathway maps, KEGG pathways present the networks of molecular interactions in cells and particular organisms. Of the 118, 043 unigenes, 54,245 had significant matches with at least one KEGG pathway in the database and were assigned to 133 KEGG pathways in total (Table 2). The most represented pathways were 'metabolic pathways' (12,473 members) and 'biosynthesis of secondary metabolites' (6980 members), followed by 'biosynthesis of antibiotics' (3268 members), 'microbial metabolism in diverse environments' (2777 members), and 'carbon metabolism' (2089 members). Additionally, 1339 unigenes were associated with the 'plant hormone signal transduction' pathway.

\section{Alternatively spliced unigenes}

The long PacBio sequencing reads can provide extensive information about alternative splicing. In this study, 27, 975 unigenes had two or more alternatively spliced isoforms, 15,074 had three or more distinct isoforms, and 10,909 had four or more distinct isoforms (Fig. 4a). Seven alternative splicing types were identified based on a SUPPA analysis, including exon skipping (938, 5.5\%), alternative $5^{\prime}$ splice site $(3044,17.8 \%)$, alternative $3^{\prime}$ splice site (3336, 19.5\%), mutually exclusive exon (305, $1.8 \%$ ), retained intron (8705 51\%), alternative first exon (646, 3.8\%), and alternative last exon $(108,0.6 \%)$. Therefore, retained intron, alternative 3 ' splice site, and alternative $5^{\prime}$ splice site were the main alternative splicing types (Fig. 4b).

\section{Comparison of the transcriptomes of normal and mutant capitula}

Unigenes common to normal and mutant capitula.

A total of 124,284 unigenes were shared by the normal and mutant capitula (Fig. 5a). In contrast, 3269 and 955 unigenes were specifically expressed in the normal and mutant capitula, respectively.

Genes differentially expressed between mutant and normal capitula.

The transcriptomes of the normal and mutant capitula were compared, and the reads were mapped to the reference transcriptome. A total of 35,419 DEGs (8232 upregulated and 27,187 down-regulated in the mutant capitula relative to the corresponding levels in the normal capitula) were identified between the normal and mutant

Table 1 De novo assembly results

\begin{tabular}{llllllll}
\hline Unigene & $\begin{array}{l}\text { N50 } \\
\text { (bp) }\end{array}$ & $\begin{array}{l}\text { Max length } \\
\text { (bp) }\end{array}$ & $\begin{array}{l}\text { Min length } \\
\text { (bp) }\end{array}$ & $\begin{array}{l}\text { Average length } \\
\text { (bp) }\end{array}$ & $\begin{array}{l}\text { Total assembled } \\
\text { bases }\end{array}$ & $\begin{array}{l}\text { GC\% Annotation } \\
\text { counts }\end{array}$ & $\begin{array}{l}\text { Annotation } \\
\text { ratio }\end{array}$ \\
\hline 130,097 & 3013 & 14,273 & 57 & 2510 & $494,377,327$ & 40.06 & 118,589 \\
\hline
\end{tabular}




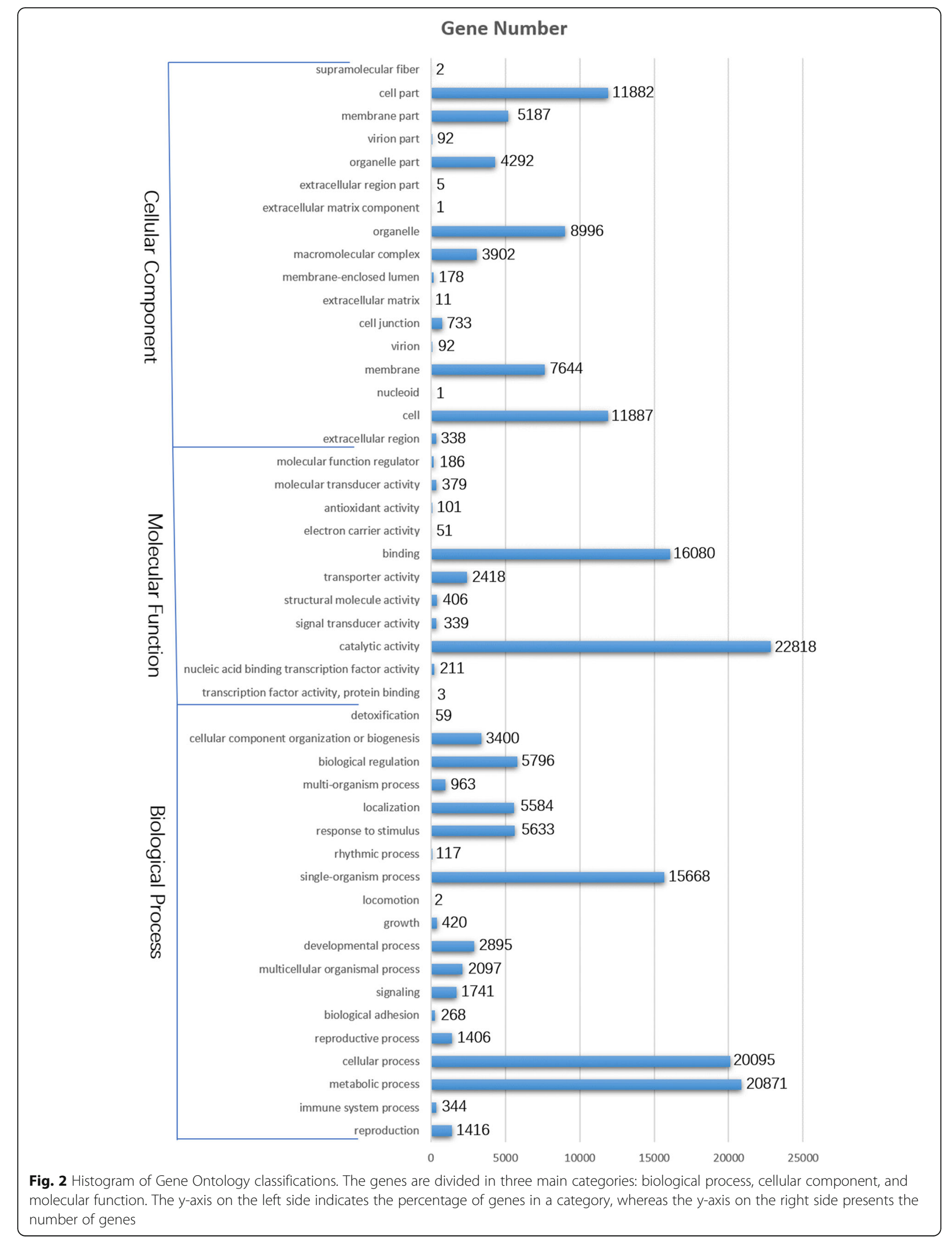




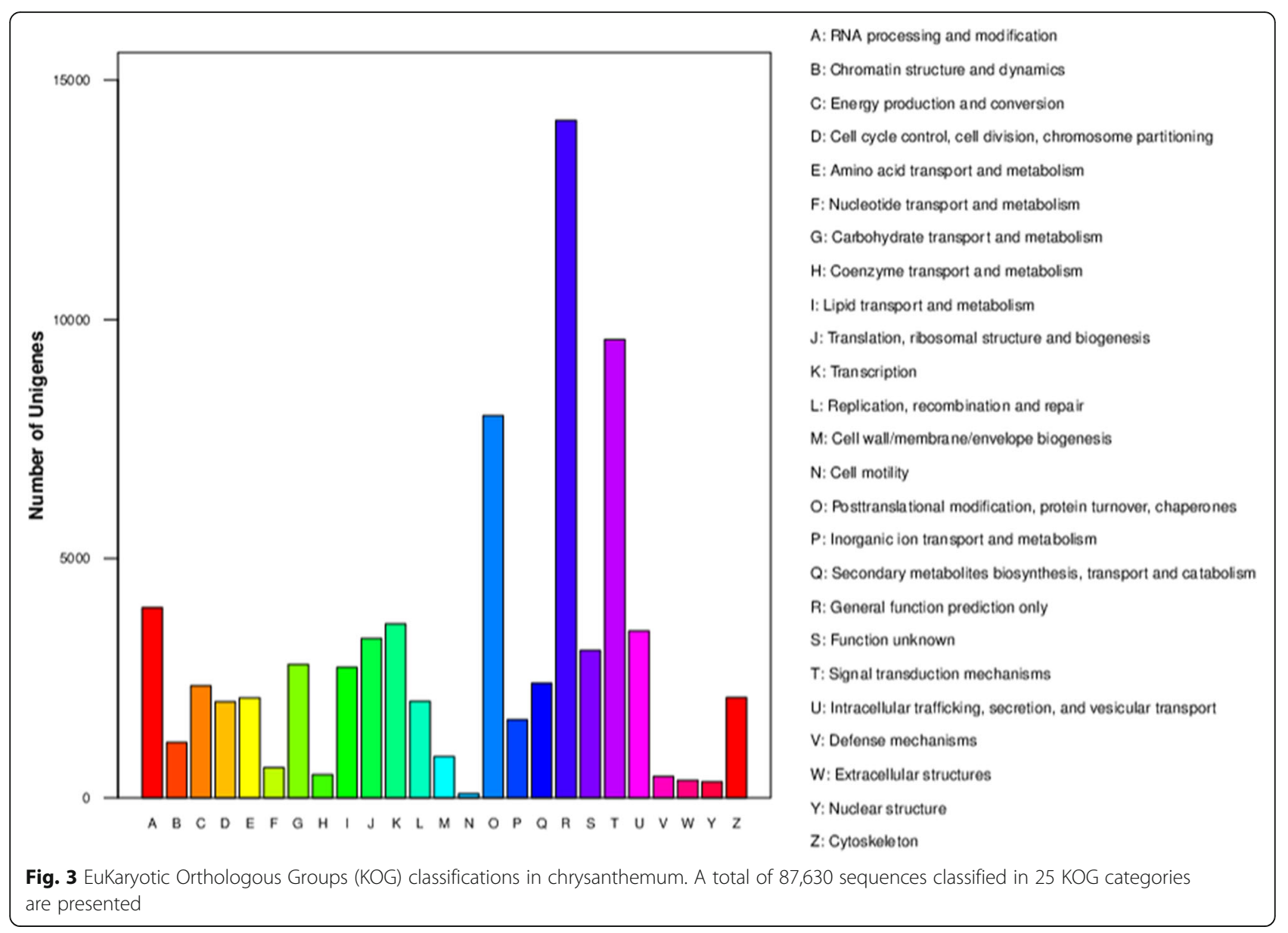

capitula (Fig. 5b). The correlation coefficient for the gene expression levels in the normal and mutant capitula was 0.8897 , which was determined using an algorithm developed from the correlation scatter plot.

A total of 131 DEGs were specifically expressed in the mutant capitula, including TCP1 and AP2/ERF domain-containing genes. Conversely, 2132 DEGs were specifically expressed in the normal capitula, including some important transcription factor genes (MYB, GRAS, and BTF3 genes), ubiquitin-conjugating enzyme genes, zinc finger protein genes, and many unannotated genes. These genes may have important functions in developing chrysanthemum flowers, especially during the pistil determination and development stage. The production of normal capitula composed of ray florets with normally developed pistils and purple corollas and mutant capitula containing ray florets with green corollas and vegetative buds may be due to significant differences in the expression of these genes. Details regarding the annotation of the DEGs specifically expressed in the mutant and normal capitula are provided in Additional files 2 and 3, respectively.
The GO and KEGG pathway enrichment analyses of the DEGs uncovered differences in biological processes and pathways between the mutant and normal capitula. The expression levels of 256 genes annotated with the 'reproduction' GO term (GO:0000003) in the biological process category were all considerably lower in the mutant capitula than in the normal capitula. Of these genes, 11 were specifically expressed in the normal capitula, including WD40 and UBA1-like protein-encoding genes. These genes may play important roles in the regulatory pathways related to chrysanthemum reproduction (Additional file 4).

A total of 6733, 7216, and 3879 DEGs were enriched in the biological process, molecular function, and cellular component categories, respectively (Additional files 5-7). In the biological process category, the main terms were 'metabolic process' (GO: 0008152, 5128 DEGs), 'cellular process' (GO:0009987, 4758 DEGs), and 'single-organism process' (GO: 0044699, 4017 DEGs). In the molecular function category, the most represented terms were 'catalytic activity' (GO: GO:0003824, 5765 DEGs), 'binding' (GO: 0005488, 3903 DEGs), and 'organic cyclic compound 
Table 2 Enriched KEGG pathways among chrysanthemum unigenes

\begin{tabular}{|c|c|c|c|}
\hline KEGG Categories & Unigene number & Rotio of no. & Pathway ID \\
\hline Metabolic pathways & 12,473 & $40.16 \%$ & ko01100 \\
\hline Biosynthesis of secondary metabolites & 6980 & $22.47 \%$ & ko01110 \\
\hline Biosynthesis of antibiotics & 3268 & $10.52 \%$ & ko01130 \\
\hline Microbial metabolism in diverse environments & 2777 & $8.94 \%$ & ko01120 \\
\hline Carbon metabolism & 2089 & $6.73 \%$ & ko01200 \\
\hline Protein processing in endoplasmic reticulum & 1858 & $5.98 \%$ & ko04141 \\
\hline Biosynthesis of amino acids & 1766 & $5.69 \%$ & ko01230 \\
\hline Spliceosome & 1725 & $5.55 \%$ & ko03040 \\
\hline Endocytosis & 1546 & $4.98 \%$ & ko04144 \\
\hline Starch and sucrose metabolism & 1511 & $4.86 \%$ & ko00500 \\
\hline RNA transport & 1350 & $4.35 \%$ & ko03013 \\
\hline Plant hormone signal transduction & 1339 & $4.31 \%$ & ko04075 \\
\hline Ubiquitin mediated proteolysis & 1205 & $3.88 \%$ & ko04120 \\
\hline Plant-pathogen interaction & 1202 & $3.87 \%$ & ko04626 \\
\hline mRNA surveillance pathway & 1085 & $3.49 \%$ & ko03015 \\
\hline Purine metabolism & 1080 & $3.48 \%$ & ko00230 \\
\hline RNA degradation & 1045 & $3.36 \%$ & ko03018 \\
\hline Ribosome & 1016 & $3.27 \%$ & ko03010 \\
\hline Oxidative phosphorylation & 1013 & $3.26 \%$ & ko00190 \\
\hline Aminoacyl-tRNA biosynthesis & 933 & $3.00 \%$ & ko00970 \\
\hline Amino sugar and nucleotide sugar metabolism & 898 & $2.89 \%$ & ko00520 \\
\hline Glycolysis / Gluconeogenesis & 811 & $2.61 \%$ & ko00010 \\
\hline Pyruvate metabolism & 799 & $2.57 \%$ & ko00620 \\
\hline Glycerophospholipid metabolism & 793 & $2.55 \%$ & ko00564 \\
\hline Pyrimidine metabolism & 790 & $2.54 \%$ & ko00240 \\
\hline Glyoxylate and dicarboxylate metabolism & 781 & $2.51 \%$ & ko00630 \\
\hline Peroxisome & 711 & $2.29 \%$ & ko04146 \\
\hline Fatty acid metabolism & 708 & $2.28 \%$ & ko01212 \\
\hline Phagosome & 699 & $2.25 \%$ & ko04145 \\
\hline alpha-Linolenic acid metabolism & 678 & $2.18 \%$ & ko00592 \\
\hline Cysteine and methionine metabolism & 667 & $2.15 \%$ & ko00270 \\
\hline Carbon fixation in photosynthetic organisms & 614 & $1.98 \%$ & ko00710 \\
\hline Glycine, serine and threonine metabolism & 583 & $1.88 \%$ & ko00260 \\
\hline Phosphatidylinositol signaling system & 556 & $1.79 \%$ & ko04070 \\
\hline Phenylpropanoid biosynthesis & 525 & $1.69 \%$ & ko00940 \\
\hline
\end{tabular}

binding' (GO:0097159, 2386 DEGs). Finally, in the cellular component category, the most common terms were 'cell' (GO:0005623, 2633 DEGs), 'cell part' (GO:0044464, 2630 DEGs), and 'intracellular' (GO:0005622, 2490 DEGs). Thus, the physiological and biochemical activities involved in metabolic, cellular, and single-organism processes differed between the mutant and normal capitula. In total, 16,342 down-regulated and 5485 up-regulated DEGs in the mutant capitula relative to the corresponding levels in the normal capitula were enriched in many KEGG pathways (Additional files 8 and 9). Interestingly, all of the DEGs enriched in the 'brassinosteroid biosynthesis' (ko00905) and 'plant hormone signal transduction' (ko04075) KEGG pathways were expressed at lower levels in the mutant capitula than in the normal capitula, implying that plant hormone signal transduction activities were suppressed in the mutant capitula. The enriched GO terms and KEGG pathways are listed in Additional files 5-9. 

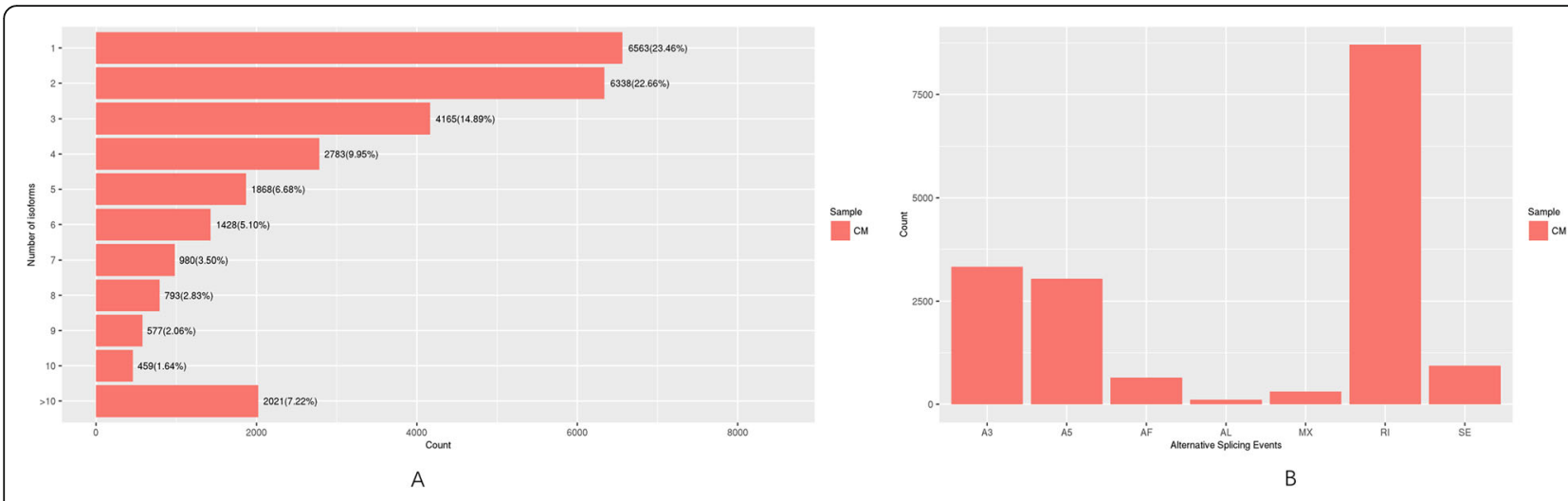

Fig. 4 Alternatively spliced genes. (A) Alternatively spliced unigenes. (B) Alternative splicing types

\section{Important transcription factors differentially expressed} between mutant and normal capitula

A total of 3921 important transcription factor genes from 52 classes were detected, among which 963 from the following transcription factor families were substantially differentially expressed between the normal and mutant capitula: AP2 (14 members), ARF (35 members), B3 (41 members), BBR-BPC (3 members), BES1 (8 members), bHLH (75 members), bZIP (22 members), C2H2 (84 members), C3H (51 members), CAMTA (2 members), CO-like (5 members), DBB (11 members), Dof (9 members), E2F/DP (4 members), ERF (98 members), FAR1 (15 members), G2-like (39 members), GATA (8 members), GeBP (1 member), GRAS (29 members), GRF (1 member), HB-other (6 members), HB-PHD (1 member), HD-ZIP (51 members), HSF (20 members), LBD (7 members), LSD (1 member), MIKC (12 members), M- type (9 members), MYB (80 members), NAC (25 members), NF-X1 (12 members), NF-YA (3 members), NFYB (8 members), NF-YC (5 members), Nin-like (40 members), S1Fa-like (6 members), SBP (13 members), SRS (2 members), TALE (17 members), TCP (3 members), Trihelix (22 members), WRKY (56 members), YABBY (1 member), and ZF-HD (8 members). More specifically, the ERF, C2H2, MYB, bHLH, and WRKY transcription factor families respectively had 98, 84, 80, 75 , and 56 members with expression levels that were extremely different between the normal and mutant capitula. Additionally, some important transcription factor genes were expressed only in the normal capitula, including $36 \mathrm{C} 2 \mathrm{H} 2$ genes, 6 bZIP genes, 5 bHLH genes, 5 MYB genes, $4 \mathrm{HB}$-other genes, $2 \mathrm{C} 3 \mathrm{H}$ genes, $2 \mathrm{E} 2 \mathrm{~F} / \mathrm{DP}$ genes, 2 GATA genes, 1 ERF gene, 1 HSF gene, 1 NF-X1 gene, 1 TALE gene, and 1 Trihelix gene.

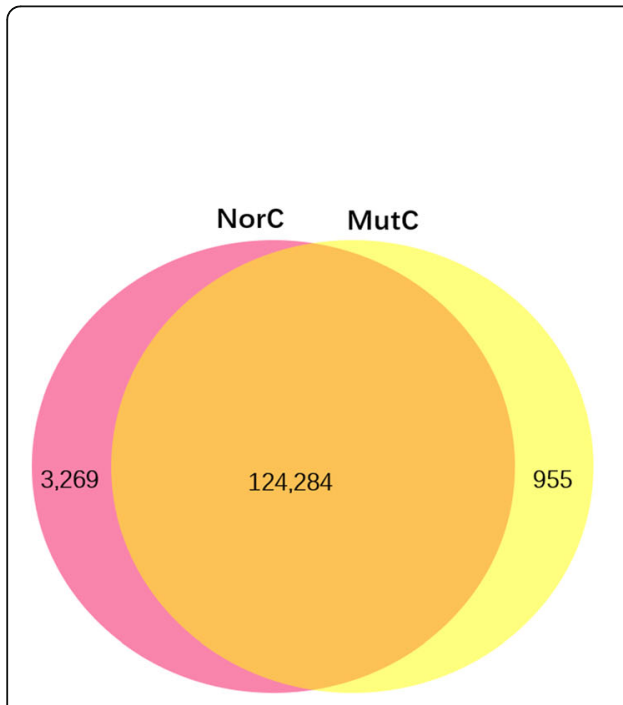

A

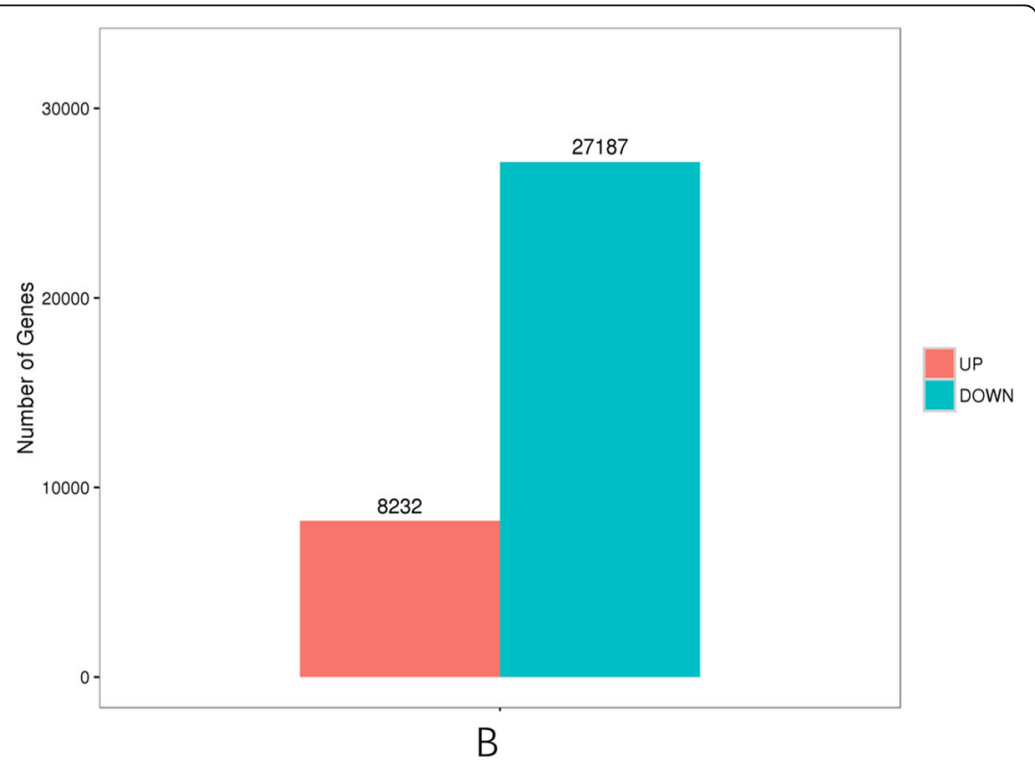

Fig. 5 Venn diagram of the number of expressed genes. (a) Venn diagram of the number of genes expressed in the normal capitula (NorC) and the mutant capitula (MutC). (b) Number of up-regulated and down-regulated genes between the normal and mutant capitula 
These results suggest that many transcription factors are important for floral development, but the functions of some transcription factors in developing flowers remain to be investigated. The important transcription factor genes substantially differentially expressed between the normal and mutant capitula may be crucial for the phenotypic variations between the normal and mutant capitula. These genes are presented in Additional file 10.

\section{Identification and expression analysis of genes involved} in the photoperiod and GA pathways in chrysanthemum As a typical short-day plant, chrysanthemum can flower in response to a single short day. Homologs of the important regulators of the photoperiod pathway in chrysanthemum were identified. Molecular genetic studies have identified many genes required for responses to the day length, with some encoding important regulators of flowering, whereas other genes encode components of the light signal transduction pathways or pathways involved in circadian signaling, including PHYTOCHROME (PHY), CRYPTOCHROME (CRY), LATE GIGANTEA (GI), and FKF1 (Flavin binding, Kelch repeat, F-box protein 1). In this study, many genes identified based on the transcriptome sequences were revealed as homologs of photoreceptor and circadian clock components involved in the photoperiod pathway (Fig. 6a). On the basis of the protein annotations of the mutant and normal capitula transcriptome sequences, many genes were identified, including several CRY1 and $C R Y 2$ homologs as well as homologs of PHYA, PHYB, FKF1, LHY, EFL1, EFL3, EFL4, TOC1, and GI. Moreover, homologs were detected for CONSTANS (CO), which is critical for the photoperiod response, and for FT (Flowering Locus T), which is targeted by CO. Many MADSbox genes are important for promoting floral meristem identity, including SHORT VEGETATIVE PHASE (SVP), SUPPRESSOR OF CONSTANS1 (SOC1), and APETALA1 (AP1). PISTILLATA (PI) is a floral organ identity gene that specifies petal and stamen identities in the A. thaliana flower [20] . Additionally, AGAMOUS (AG) interacts with LEAFY (LFY) and TERMINAL FLOWER1 (TFL1) to maintain the identity of an existing floral meristem [21]. We identified homologs of these MADSbox genes. APETALA2 (AP2) encodes an important promoter of floral meristem identity. Two $A P 2$ homologs were identified. $L E A F Y(L F Y)$, which is vital for the regulation of floral meristem identity, is initially expressed very early throughout the presumptive floral meristem. We did not detect the expression of $L F Y$ homologs in the mutant and normal ' $\mathrm{ZY}$ ' capitula, probably because these genes were no longer expressed at the full-bloom stage of the capitula. The SOC1 homolog identified in this study encodes an upstream regulator of $L F Y$ expression. Interestingly, its expression level was significantly higher in the mutant capitula than in the normal capitula. As an A-class-like gene, $A P 1$ expression is directly activated by LFY $[22,23]$. The AP1 homologs identified

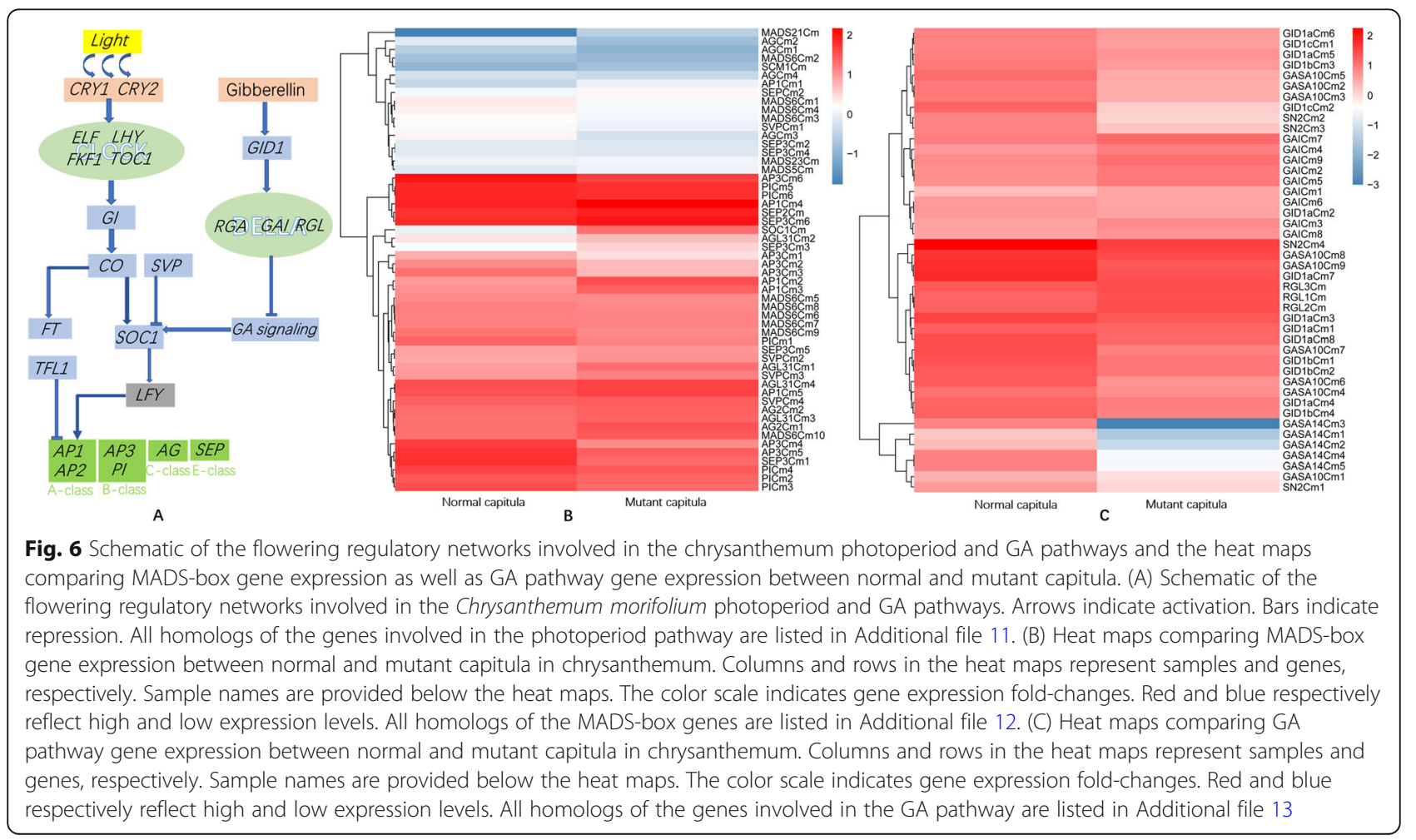


in this study were all more highly expressed in the mutant capitula than in the normal capitula.

Another A-class gene, $A P 2$, is not a MADS-box gene and it encodes a transcription factor in the AP2/EREBP family. Two AP2 homologs were identified, both of which were more highly expressed in the normal capitula than in the mutant capitula. Most core eudicot species include three distinct B-class gene lineages: PI, euAP3, and TM6; however, TM6-like genes seem to have been lost in Arabidopsis and Antirrhinum species [24]. In the current study, we identified PI and AP3 homologs, but the expression of the TM6-like genes was undetectable. In contrast, TM6-like gene expression was detected in chrysanthemums in earlier studies [25, 26]. We also identified homologs of the C-class gene $A G$ and E-like MADS-box genes in this study. The A-, B-, C-, and Elike genes were all expressed in the mutant capitula, which lacked normal stamens and pistils. Details regarding the annotation of the important genes involved in the photoperiod pathway are provided in Additional file 11.

A comparison of the expression of the detected MADS-box genes between the mutant and normal capitula revealed that the expression levels of many $A P 1$, $S E P$, and $A G L$ homologs were slightly higher in the mutant capitula than in the normal capitula. In contrast, the AP3 and PI homologs were expressed at lower levels in the mutant capitula than in the normal capitula, with AP3 homolog expression in the mutant capitula less than half of that in the normal capitula (Fig. 6b). This finding may provide researchers with an important clue regarding the molecular mechanism underlying the phenotypic variations between normal and mutant capitula. Details regarding the annotation of the MADS-box genes are provided in Additional file 12.

Previous research proved that GAs, sugars, and light help regulate various pathways required to complete the flower development process [8] . The circadian clock is affected by GA signaling, which is controlled by the transcriptional regulation of two GAINSENSITIVE DWARF1 (GID1) GA receptor genes (GID1a and GID1b) in A. thaliana [27]. Earlier studies demonstrated that GA promotes $A$. thaliana petal, stamen, and anther development by inhibiting the function of the DELLA proteins encoded by REPRESSOR OF ga1-3 (RGA), GAINSENSITIVE (GAI), RGA-LIKE1 (RGL1), RGL2, and RGL3. The GID1a, GID1b, and GID1c genes of A. thaliana have been identified [28]. The expression of GASA genes is up-regulated by GA and down-regulated by the DELLA proteins GAI and RGA, which are involved in stem elongation or floral development [29]. In this study, we identified homologs of DELLA protein-encoding genes ( $R G A, G A I$, and $R G L$ ) as well as GID1 (GID1a, GID1b, and GID1c), and GASA (GASA10 and GASA14) genes. Most of the RGA, GAI, RGL1, RGL2, and RGL3 homolog expression levels were significantly higher in the mutant capitula than in the normal capitula. Additionally, the homologs of GA receptor genes (GID1a, GID1b, and GID1c) and GA-regulated protein-encoding genes (GASA10 and GASA14) were expressed at lower levels in the mutant capitula than in the normal capitula (Fig. 6c). Therefore, the GA signaling pathway was probably suppressed in the mutant capitula. Details regarding the annotation of the important genes involved in the GA pathway are provided in Additional file 13.

\section{Identification and analysis of important regulatory and functional genes in the anthocyanin biosynthesis pathway and the pigments in the corollas of chrysanthemum florets}

The MYB-bHLH-WD40 (MBW) activator complexes modulate the expression of downstream genes required for flavonoid biosynthesis in plants. These complexes are composed of R2R3 MYB transcription factors (MYB), the basic helix-loop-helix (bHLH) transcription factors [e.g., Glabra 3 (GL3), Transparent Testa 8 (TT8), and Enhancer of Glabra3 (EGL3)], and the WD40-repeat protein TRANSPARENT TESTA GLABRA1 (TTG1) [30]. The MBW activator complexes directly mediate the expression of late anthocyanin biosynthetic genes, including chalcone isomerase $(\mathrm{CHI})$, flavonoid 3'-hydroxylase $\left(F 3^{\prime} H\right)$, dihydroflavonol reductase $(D F R)$, and anthocyanin synthase (ANS) genes, leading to the accumulation of anthocyanins [31].

To explore the molecular basis of the flower color differences between the normal and mutant capitula, we identified and analyzed the expression of genes encoding the R2R3 MYB, bHLH, and WD40-repeat proteins, including the homologs of MYB113, MYB114, MYB305, MYB46, Glabra 2 (GL2), Transparent Testa 12 (TT12), and TTG1. The expression levels of most of the R2R3 MYB genes were significantly down-regulated in the mutant capitula, with some genes not expressed at all (Fig. 7a, Additional file 14). Similarly, the expression levels of the bHLH and WD40-repeat protein genes (GL2, TT12, and TTG1) were also considerably downregulated in the mutant capitula. Hua Li et al. suggested that MdMYB8 contributes to the regulation of flavonoid biosynthesis, with the overexpression of MdMYB8 promoting flavonol biosynthesis in crabapple [32]. In this study, four MYB8-like genes (MYB8Cm1, MYB8Cm2, $M Y B 8 C \mathrm{~m} 3$, and $M Y B 8 C m 4$ ) were not expressed in the mutant capitula lacking anthocyanins; the lack of expression was confirmed by quantitative real-time PCR (qRTPCR). Flavonol is an upstream substrate for anthocyanin biosynthesis. Therefore, this result implied these four MYB8-like genes may encode important regulators of 


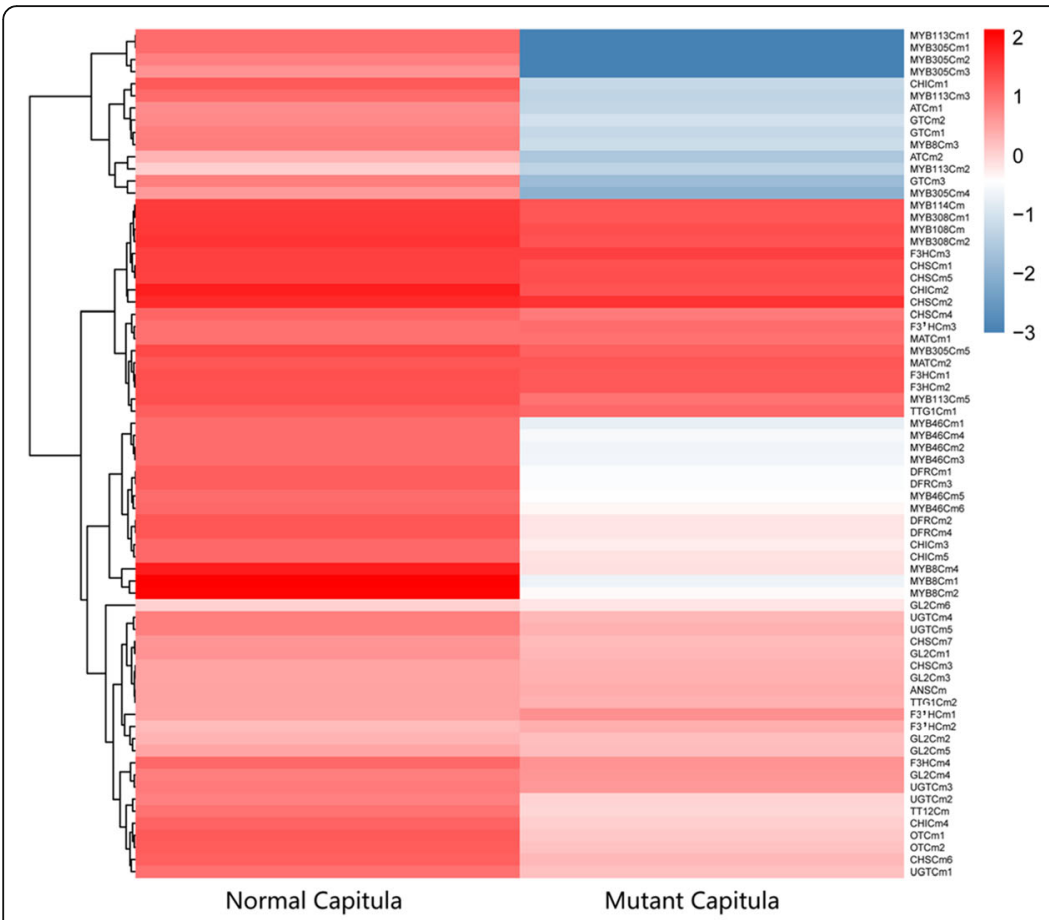

A

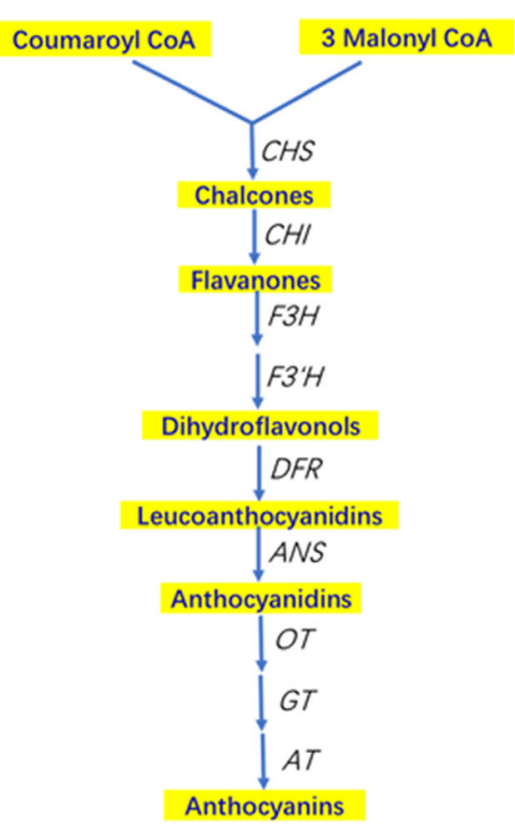

B

Fig. 7 Heat maps comparing anthocyanin biosynthesis pathway gene expression between normal and mutant capitula as well as the anthocyanin biosynthesis pathway and identified regulatory genes. (A) Heat maps comparing anthocyanin biosynthesis pathway gene expression between normal and mutant capitula in chrysanthemum. Columns and rows in the heat maps represent samples and genes, respectively. Sample names are provided below the heat maps. The color scale indicates gene expression fold-changes. Red and blue respectively reflect high and low expression levels. (B) Anthocyanin biosynthesis pathway and the regulatory genes identified based on the chrysanthemum transcriptome. CHS: chalcone synthase, CHI: chalcone isomerase, F3H: flavanone 3-hydroxylase, F3'H: flavonoid 3'-hydroxylase, DFR: dihydroflavonol 4-reductase, ANS: anthocyanidin synthase, OT: 3-O-glucoside-6"-O-malonyltransferase, GT: glucosyltransferase, AT: acyltransferase. The genes in the anthocyanin biosynthesis pathway are listed in Additional file 14

anthocyanin biosynthesis in the corollas of chrysanthemum florets.

The key functional genes in the anthocyanin biosynthesis pathway, including genes encoding chalcone synthase, chalcone flavonone isomerase, flavanone 3hydroxylase, flavonoid 3'-hydroxylase, dihydroflavonol 4-reductase, glucosyltransferase, 3-O-glucoside-6"-Omalonyltransferase, acyltransferase, and anthocyanidin synthase, were identified based on the transcriptome (Fig. 7b). Most of the late anthocyanin biosynthetic genes were expressed at lower levels in the mutant capitula than in the normal capitula (Fig. 7a).

Interestingly, all four DFR homologs and one CHI homolog were expressed at extremely low levels (close to 0 ) in the mutant capitula. A qRT-PCR analysis indicated that the $C H I$ homolog was not expressed in the mutant capitula, but was expressed in the normal capitula. Information regarding the annotation of these genes in the anthocyanin biosynthesis pathway is provided in Additional file 14.

The pigment types and contents determine the diversity in flower colors. A qualitative analysis of the pigments in the florets of normal and mutant capitula was performed using an HPLC system. Anthocyanins were detected in the florets of normal capitula, but not in the florets of mutant capitula (Fig. 8b). The detected anthocyanins were mainly delphinidin, cyanidin, petunidin, pelargonidin, peonidin, and malvidin (Fig. 8a). Accordingly, the down-regulated expression of genes encoding MBW activator complex components inhibited the expression of late anthocyanin biosynthetic genes, including $C H I$ and DFR, leading to an anthocyanin deficiency in the mutant capitula.

\section{Verification of gene expression profiles in qRT-PCR assays}

To further verify the expression profiles of the unigenes revealed following the Illumina sequencing analyses, 16 unigenes were selected for a qRT-PCR analysis of the mutant and normal capitula originally used for the RNA-seq experiment. Four $M Y B$-like genes ( $M Y B 8 C m 1$, $M Y B 8 C m 2, M Y B 8 C m 3$, and $M Y B 8 C m 4)$ and one $C H I$ gene $(\mathrm{CHICZ}-1)$ were selected because of their important regulatory effects on anthocyanin biosynthesis. The other analyzed genes were selected randomly, including 

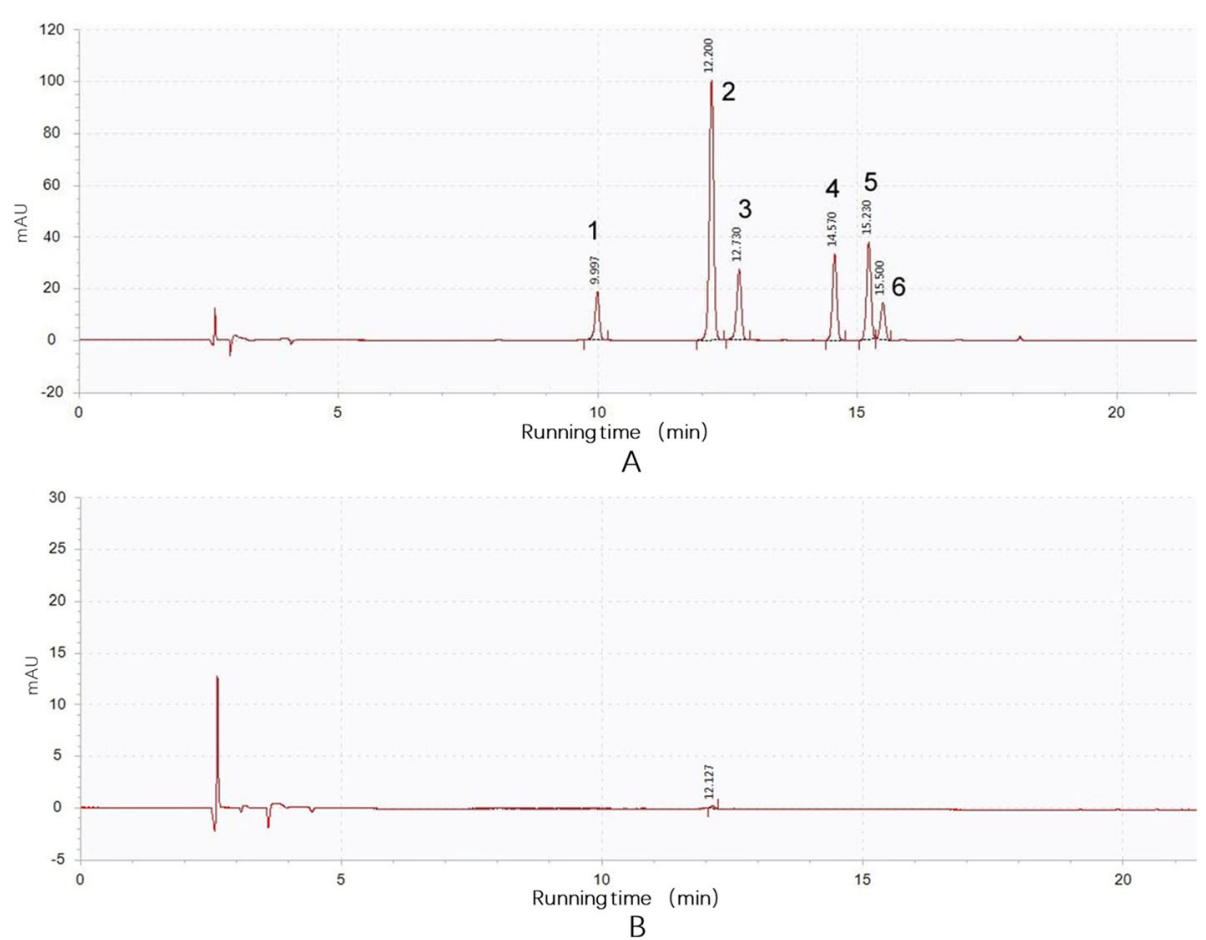

Fig. 8 Qualitative analysis of the pigments in the normal (A) and mutant (B) capitula. 1. delphinidin; 2. cyanidin; 3. petunidin; 4. pelargonidin; 5. peonidin; 6. malvidin

one COP1 gene (COP1CZ), one EF2 gene (EF2CZ) specifically expressed in the normal capitula, one histone $H 2 B$ gene (HIS2BCZ), one catalase gene (CAT3CZ), one photosystem II $5 \mathrm{kDa}$ protein gene (PSBTCZ), one annexin D8 gene (ANN2CZ), one arginine kinase gene ( $A R G K C Z$ ), and four unigenes encoding uncharacterized proteins (UnknownCZ1, UnknownCZ2, UnknownCZ3, and UnknownCZ4). The resulting data for all 16 genes were consistent with the sequencing data (Additional file 15, Fig. 9).

\section{Discussion}

The normal and mutant capitula differed primarily in the color of ray floret corollas (the normal and mutant capitula were purple and green, respectively) and the replacement of the ray floret pistils of the normal capitula with vegetative buds in the mutant capitula. A wholetranscriptome analysis of the DEGs in the mutant and normal capitula reflected the complexity of the regulatory machinery underlying the phenotypic differences between the capitula. A qualitative analysis of pigments

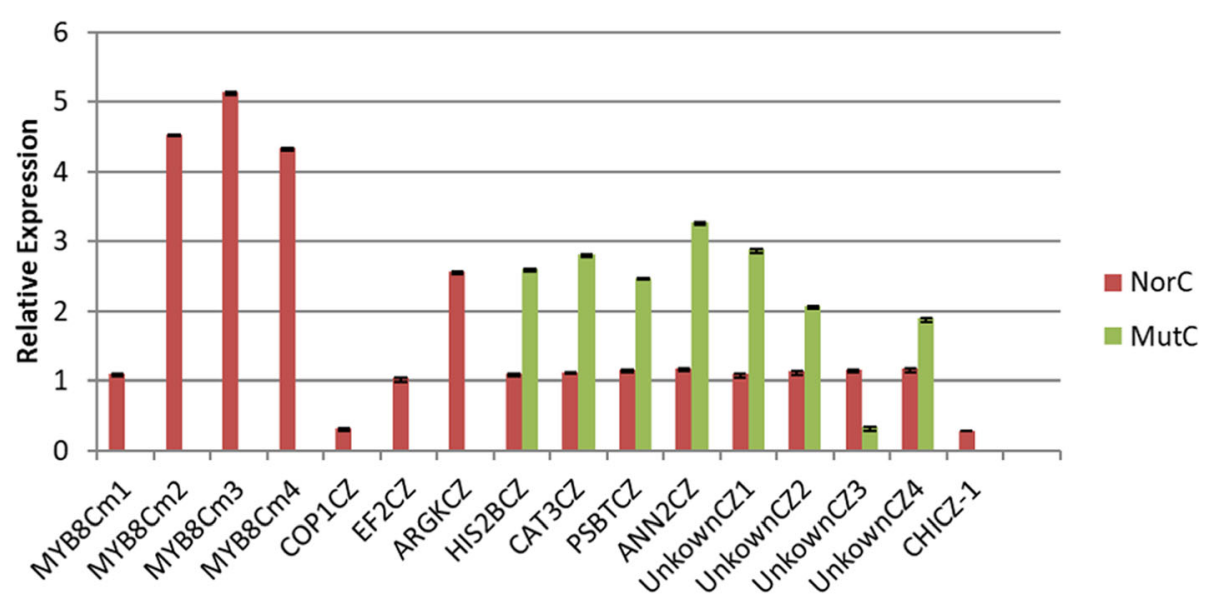

Fig. 9 Expression profiles of 16 genes in Chrysanthemum morifolium revealed by qRT-PCR 
revealed anthocyanins were not synthesized and did not accumulate in the florets of the mutant capitula. A gene expression analysis indicated the down-regulated expression of MBW activator complex genes inhibited the expression of late anthocyanin biosynthetic genes, leading to the deficiency of anthocyanins in the mutant capitula. Furthermore, as one of the major flower pigments in higher plants, the synthesis and accumulation of anthocyanins is an integral part of flower development in most plant species and is tightly linked with petal cell expansion. The activation of the anthocyanin biosynthesis pathway during petal development requires both environmental and endogenous signals, but the endogenous regulatory system is the main factor controlling anthocyanin biosynthesis in developing flowers [8]. Therefore, the anthocyanin deficiency in the mutant capitula is likely related to the mutation causing the ray floret pistils to be replaced by vegetative buds. This mutation may be associated with the differences in the expression of important transcription factor genes and phytohormone signaling pathway genes between the normal and mutant capitula.

\section{The anthocyanin deficiency in the mutant capitula may} be related to a mutation to the ray floret pistils that may be required for anthocyanin biosynthesis in the corollas In Petunia hybrida, GAs, sugars, and light are required for inducing the transcription of anthocyanin biosynthesis genes and the accumulation of pigments in developing corollas [25]. An earlier study proved that in the initial stages of $P$. hybrida flower development, GAs produced by the anthers control anthocyanin biosynthesis and accumulation in the corollas by activating the transcription of specific anthocyanin biosynthesis pathway genes, and that the removal of stamens during the early flower development stage inhibits anthocyanin biosynthesis in the corollas [33]. Tiancong Qi et al. revealed GA and jasmonate coordinately activate the MBW complex by inducing the degradation of DELLA proteins and JASMONATE ZIM-domain proteins [34]. A study by G. W. M. Barendse et al. confirmed that in P. hybrida and Lilium species, unpollinated styles and ovaries contain GA, with more GA in the ovaries than in the styles [35].

In C. morifolium, the capitula of many varieties (e.g., 'Ping Pong') have only ray florets because of the extensive breeding for the double-flowered trait. Interestingly, most of the capitula in C. morifolium ' $\mathrm{ZY}$ ' comprise ray florets, but no disk florets. In this study, a comparison of the GA signaling pathway gene expression between the normal and mutant capitula indicated that the GA signaling pathway was probably suppressed in the mutant capitula because of a lack of normal pistils, which likely prevented the mutant capitula from synthesizing anthocyanins. Therefore, we hypothesized that when the pistils of ray florets are mutated and replaced by vegetative buds, the anthocyanin biosynthesis in the corollas is blocked. Consequently, normal pistils may be required for the anthocyanin biosynthesis in chrysanthemums.

\section{Anthocyanin deficiency may also be related to the down- regulated expression of the B-class MADS-box genes AP3 and $P I$ in the mutant capitula}

Katsutomo Sasaki et al. revealed the synergistic effects of the proteins encoded by PI and AP3 homologs (TfGLO and $T f D E F)$ in torenia plants. Anthocyanins accumulate similarly in the sepals and petals of TfGLO-overexpressing plants, which produce purple-stained sepals. Plants in which TfGLO expression is suppressed have flowers with serrated petals, whereas plants with suppressed TfDEF expression produce flowers with partially decolorized petals. Both TfGLO- and TfDEF-suppressed plants have some sepal-like cells at their petal surfaces [36]. Accordingly, the B-class MADS-box genes AP3 and PI may be involved in anthocyanin biosynthesis. In the current study, we determined that the AP3 and PI homologs were expressed at relatively low levels in the mutant capitula. More specifically, AP3 homolog expression in the mutant capitula was less than half of that in the normal capitula. Additionally, anthocyanins did not accumulate in the mutant capitula. Therefore, the anthocyanin deficiency of the mutant capitula may be related to the down-regulated expression of the B-class MADSbox genes $A P 3$ and $P I$.

\section{Protective mechanisms may be disrupted in mutant inflorescences because of a repressed jasmonate pathway, which prevents the activation of anthocyanin biosynthesis}

Plants have developed constitutive and inducible defenses against pests and pathogens. The inducible defenses depend on the combined effects of jasmonate and ethylene [37]. In this study, the expression levels of the DEGs associated with the KEGG pathway 'plant hormone signal transduction' (ko04075) were significantly lower in the mutant capitula than in the normal capitula. These DEGs included jasmonate response factor genes and ethylene-responsive sensor genes. Additionally, 'defense mechanisms' $(673,0.77 \%)$ was one of the smallest groups among the 25 KOG categories. Therefore, the protective mechanisms in mutant inflorescences may be inhibited because of a repressed jasmonate pathway.

Jasmonate up-regulates the expression of several anthocyanin biosynthetic genes essential for anthocyanin accumulation [38]. Zhihong Peng et al. reported that jasmonate-induced anthocyanin accumulation is suppressed by a brassinosteroid deficiency and blocked BR signaling. Brassinosteroids enhance jasmonate-induced anthocyanin accumulation in A. thaliana seedlings by 
regulating the expression of late anthocyanin biosynthetic genes (e.g., DFR, LDOX, and UF3GT) [39]. In the current study, the DEGs associated with the KEGG pathway 'brassinosteroid biosynthesis' (ko00905) were expressed at lower levels in the mutant capitula than in the normal capitula, with the expression of some of the DEGs almost undetectable in the mutant capitula. Similarly, the expression levels of four DFR homologs were very low in the mutant capitula. Thus, anthocyanin biosynthesis is not activated probably because of the suppressed jasmonate pathway due to the brassinosteroid deficiency.

\section{The transcription factor genes expressed in the normal capitula, but not in the mutant capitula, may be associated with the mutation to the pistils of ray florets} In this study, 963 of the 3921 detected important transcription factor genes were substantially differentially expressed between the normal and mutant capitula, including members of the ERF, C2H2, MYB, bHLH, and WRKY transcription factor families. Interestingly, some transcription factor genes were expressed exclusively in the normal capitula, including $35 \mathrm{C} 2 \mathrm{H} 2$ genes, $7 \mathrm{MYB}$ genes, 6 bZIP genes, 6 bHLH genes, 4 HB-other genes, 2 $\mathrm{C} 3 \mathrm{H}$ genes, $2 \mathrm{E} 2 \mathrm{~F} / \mathrm{DP}$ genes, 2 GATA genes, 1 ERF gene, 1 HSF gene, 1 NF-X1 gene, 1 TALE gene, and 1 Trihelix gene. Of these genes, the lack of expression of four MYB genes (MYB8Cm1, MYB8Cm2, MYB8Cm3, and $M Y B 8 C m 4)$ in the mutant capitula was confirmed by qRT-PCR.

The $\mathrm{C} 2 \mathrm{H} 2$ zinc finger transcription factors play important roles in many biological processes related to plant growth and development, hormone signaling, and stress responses [40]. In many plants, the $\mathrm{C} 2 \mathrm{H} 2$ zinc finger proteins influence the tolerance to salt, cold, drought, and oxidative stresses as well as responses to light stress and pathogens [40]. Additionally, some members help regulate floral development. For example, SIZF2 affects flower and leaf shapes in A. thaliana. Previous investigations proved that the protein encoded by the SUPERMAN (SUP) gene determines the boundary between the stamen and carpel whorls, suppresses Bclass gene expression, and promotes stem cell termination in the fourth whorl of A. thaliana flowers [40-42] - In the present study, $35 \mathrm{C} 2 \mathrm{H} 2$ genes were not expressed in the mutant capitula, implying the encoded zinc finger transcription factors may be important for pistil development or the anthocyanin biosynthesis and accumulation in the chrysanthemum ray floret corollas.

The basic leucine zipper (bZIP) transcription factors form one of the largest transcription factor families and are critical for controlling plant development and stress responses [43]. These transcription factors are reportedly involved in various floral developmental processes in plants, including pollen development as well as floral transitions and initiation [44, 45]. In the current study, the deficient expression of six bZIP genes in the mutant capitula suggests that the encoded transcription factors are important regulators of chrysanthemum ray floret development.

The MYB transcription factors, which belong to one of the largest and most diverse transcription factor families in the plant kingdom, are common among eukaryotes and play essential roles in diverse physiological and biochemical processes controlling plant growth and development [46, 47]. The bHLH transcription factors, which possess a highly conserved bHLH domain that includes a basic region and an HLH region, are crucial for plant growth and development, metabolic regulation, and responses to environmental changes. The regulatory functions of bHLH transcription factors related to active secondary metabolism, especially anthocyanin biosynthesis, have been a topic of interest among researchers [48]. We observed that several bHLH and MYB genes were not expressed in the mutant capitula, suggesting these genes are essential for regulating normal ray floret development in chrysanthemums. Accordingly, these genes should be more thoroughly functionally characterized. The other transcription factor genes that were not expressed in the mutant capitula may also encode important regulators of chrysanthemum ray floret growth and development, including the $\mathrm{HB}$ genes, two $\mathrm{C} 3 \mathrm{H}$ genes, two E2F/DP genes, two GATA genes, one ERF gene, one HSF gene, one NF-X1 gene, one TALE gene, and one Trihelix gene. The mutation resulting in the production of vegetative buds in place of the ray floret pistils may be associated with the lack of expression of these transcription factor genes in the mutant capitula. Consequently, the regulatory functions of the transcription factors in developing chrysanthemum flowers should be explored in detail in the future.

\section{Conclusions}

In this study, a comparative transcriptome analysis revealed significant differences in gene expression and signaling pathways between the mutant and normal capitula. The identified DEGs included important regulators of the phenotypic differences between the normal and mutant capitula. Additionally, the transcription factor genes and the genes associated with the photoperiod and GA pathways, floral organ identity, and the anthocyanin biosynthesis pathway were differentially transcribed between the normal and mutant capitula. A qualitative analysis of the pigments in the florets of normal and mutant capitula revealed anthocyanins were synthesized and accumulated only in the florets of the normal capitula. Therefore, the anthocyanin deficiency in the mutant capitula may be related to the mutation of 
ray floret pistils and their replacement by vegetative buds. Moreover, pistils may be required for the anthocyanin biosynthesis in the corollas of chrysanthemum ray florets. Furthermore, the transcription factor genes expressed in the normal capitula, but not in the mutant capitula, may also be associated with the mutation to the ray floret pistils. The transcriptome analysis described herein provided valuable information regarding the molecular mechanisms underlying the production of normal ray florets with pistils and purple corollas as well as mutant ray florets with green corollas and vegetative buds in C. morifolium. The results of this study may be useful for developing enhanced techniques for studying the regulation of flower shapes and colors and for breeding novel chrysanthemum varieties.

\section{Methods}

\section{Plant materials and RNA extraction}

The normal and mutant capitula used in this study were obtained from a cut-flower chrysanthemum variety $(C$. morifolium ' $\mathrm{ZY}$ ', a hybrid of chrysanthemum varieties) cultivated in a greenhouse under an 8-h light/16-h dark cycle at $23^{\circ} \mathrm{C}$ at the Beijing Academy of Agriculture and Forestry Sciences $\left(116.3^{\circ} \mathrm{E}, 39.9^{\circ} \mathrm{N}\right)$. After the florets of the capitula were fully formed, about 3-6 normal capitula, 3-6 mutant capitula, 3-4 fully expanded leaves, 1$2 \mathrm{~g}$ roots, and $1-2 \mathrm{~g}$ stems were collected between 9:00 and 12:00 pm. Three biological replicates were collected for each sample. The collected samples were frozen immediately in liquid $\mathrm{N}_{2}$ and stored at $-80^{\circ} \mathrm{C}$. Total RNA was extracted from the frozen samples using the RNeasy Plant Mini Kit (Qiagen, China). The RNA was quantified and the quality was assessed using a NanoDrop ND2000 spectrophotometer (Thermo Scientific).

\section{Library construction, PacBio sequencing, and data processing}

Sequencing libraries were constructed and sequenced with the PacBio Sequel system (PacBio, CA, USA). Briefly, total RNA $(1 \mu \mathrm{g})$ from each of the five tissues was pooled, after which the mRNA was enriched using oligo (dT) magnetic beads. The enriched mRNA was reverse transcribed into cDNA with the Clontech AMSR Ter PCR cDNA Synthesis Kit. After a PCR amplification, the BluePippin Size Selection System was used to produce two bins: $0.5-2$ and 2-6 kb. A large-scale PCR was performed and the cDNA products were used to construct SMRTbell template libraries with the SMRTbell Template Prep Kit. The SMRTbell templates were annealed to sequencing primers and bound to polymerase, after which they were sequenced on the PacBio Sequel platform using P6-C4 chemistry with 10-h movies by Gene Denovo Biotechnology Co.
The raw sequencing reads were classified and clustered into consensus transcripts using the PacBio IsoSeq pipeline (https://github.com/PacificBiosciences/IsoSeq SA3nUP) and the SMRT Analysis software suite (https:// www.pacb.com/support/software-downloads/). Briefly, circular consensus sequence (CCS) reads were extracted from the subreads BAM file. The CCS reads were classified into four categories: full-length (FL) non-chimeric, FL chimeric, non-FL, and short reads based on the cDNA primers and poly(A) tail signals. The short reads were discarded. The FL non-chimeric reads were clustered using the Iterative Clustering for Error Correction (ICE) software to generate cluster consensus unigenes. Two strategies were employed to improve the accuracy of the PacBio reads. First, the non-FL reads were used to polish the cluster consensus unigenes with the Quiver software to obtain FL-polished high-quality consensus sequences (accuracy $\geq 99 \%$ ). Second, the low-quality unigenes were corrected using the Illumina short reads obtained for the same samples with the LoRDEC tool (version 0.8) [49]. Then, the final transcriptome unigene sequences were filtered by removing the redundant sequences with the CD-HIT (version 4.6.7) software (sequence identity threshold of 0.99).

\section{Illumina sequencing}

The total RNA isolated from the normal and mutant capitula was used for Illumina sequencing on an Illumina HiSeq $^{\text {ma }} 2000$ system (Illumina, San Diego, CA, USA). We purified the poly(A) mRNAs, fragmented them into small pieces, and then synthesized the first- and secondstrand cDNAs.

The double-stranded cDNAs were purified and resolved for repairing ends and adding a poly(A) tail. Sequencing adapters were then annealed to the short fragments. Briefly, a cDNA library with average insert sizes of 300-500 bp was created and sequenced using the Illumina HiSeq ${ }^{\text {Tw }} 2000$ system to generate $100 \mathrm{bp}$ paired-end reads.

\section{Basic annotation of unigenes}

Unigenes were functionally annotated on the basis of a BLASTX alignment with sequences in the following databases (E-value of $1.00 \mathrm{E}^{-5}$ ): $\mathrm{nr}$ (NCBI), Swiss-Prot (http://www.expasy.ch/sprot), KEGG (http://www. genome.jp/kegg), and KOG (http://www.ncbi.nlm.nih. gov/KOG) databases. The optimal alignment results determined the sequence direction of the unigenes. The Blast2GO program was used for the GO annotation of unigenes [50]. Unigenes with the 20 highest scores and no fewer than 33 high-scoring segment pair hits were selected for the Blast2GO analysis. The unigenes were functionally classified using the WEGO software [51]. 


\section{Analysis of chrysanthemum transcriptome sequencing results}

The number of reads per kilobase of exon model per million mapped reads (RPKM) following the RNA-seq analysis was used to calculate the expression level of each unigene [52]. The chrysanthemum transcriptome was used as a reference to screen and analyze DEGs. A rigorous algorithm was created based on the method of Audic et al. to screen the DEGs [53]. The false discovery rate (FDR) was used to affirm the $P$-value threshold in multiple tests and analyses [54]. An absolute value of the $\log _{2}$ (ratio) $\geq 2$ and FDR $<0.05$ were applied as the thresholds for determining significant differences in gene expression [55]. Only the DEGs with at least a 2-fold change in expression level were used for the differential gene expression analysis.

\section{Alternative splicing detection}

To analyze alternative splicing events in the unigenes, the coding genome reconstruction tool (Cogent) was employed to partition the transcripts into gene families based on the $k$-mer similarity, after which each family was reconstructed into a coding reference genome using De Bruijn graphs [56]. The alternative splicing events of the unigenes were analyzed using the SUPPA tool [57].

\section{Gene expression analysis based on qRT-PCR}

Total RNA was extracted from the normal and mutant capitula as described above. The total RNA was treated with DNase (Promega, USA) and then used as the template to synthesize cDNA with a reverse transcription system (Tiangen, China). A qRT-PCR analysis was completed using the PikoReal Real-Time PCR system (Thermo Fisher Scientific, Germany). Each reaction was carried out in a total volume of $20 \mu \mathrm{L}$, with $2 \mu \mathrm{L}$ firststrand cDNA as the template. The PCR program was as follows: $95^{\circ} \mathrm{C}$ for $30 \mathrm{~s} ; 40$ cycles of $95^{\circ} \mathrm{C}$ for $5 \mathrm{~s}$ and $60{ }^{\circ} \mathrm{C}$ for $30 \mathrm{~s}$. The gene-specific qRT-PCR primers listed in Additional file 13 were used to determine the relative expression level of each gene. All samples were analyzed in triplicate and the qRT-PCR experiments were performed with three biological replicates. The relative expression levels were calculated using the $2^{-\Delta \Delta \mathrm{Ct}}$ method, with the C. morifolium protein phosphatase 2A (PP2AC) gene serving as the reference control [58].

\section{Qualitative analysis of pigments in chrysanthemum flowers}

The anthocyanin profiles in the normal and mutant capitula were analyzed by HPLC. A 1.0-10.0 g sample was ground into a fine powder in liquid $\mathrm{N}_{2}$ and homogenized via a sonication at $20^{\circ} \mathrm{C}$ for $30 \mathrm{~min}$ to produce $50-\mathrm{mL}$ anthocyanin extracts [ethyl alcohol: distilled water: hydrochloric acid $(2,1: 1, \mathrm{v} / \mathrm{v} / \mathrm{v})]$ [59]. The extracts were heated in boiling water for $1 \mathrm{~h}$ and then centrifuged at $16,000 \times \mathrm{g}$ for $10 \mathrm{~min}$ at $20^{\circ} \mathrm{C}$. The supernatant was passed through a $0.45 \mu \mathrm{m}$ reinforced nylon membrane filter and then injected into an X Bridge BEH C18 column $(250 \mathrm{~mm} \times 4.6 \mathrm{~mm} \times 5 \mu \mathrm{m})$, which was used to separate the anthocyanins and flavonols. The column was maintained at $35^{\circ} \mathrm{C}$ and water containing $1 \%(\mathrm{v} / \mathrm{v})$ formic acid (A) and 1\% (v/v) acetonitrile (B) was used as the mobile phase. A gradient elution was applied at a flow rate of $0.8 \mathrm{~mL} / \mathrm{min}$ with the following conditions: $92 \% \mathrm{~A}+8 \% \mathrm{~B}, 0 \mathrm{~min} ; 88 \% \mathrm{~A}+12 \% \mathrm{~B}, 2 \mathrm{~min} ; 82 \% \mathrm{~A}+$ $18 \%$ B, 5 min; $80 \% \mathrm{~A}+20 \% \mathrm{~B}, 10 \mathrm{~min} ; 75 \% \mathrm{~A}+25 \% \mathrm{~B}$, 12 min; $70 \% \mathrm{~A}+30 \% \mathrm{~B}, 15 \mathrm{~min} ; 55 \% \mathrm{~A}+45 \% \mathrm{~B}, 18 \mathrm{~min}$; $20 \% \mathrm{~A}+80 \% \mathrm{~B}, 20 \mathrm{~min} ; 92 \% \mathrm{~A}+8 \% \mathrm{~B}, 22 \mathrm{~min} ; 92 \% \mathrm{~A}+$ $8 \% \mathrm{~B}, 30 \mathrm{~min}$. The injection volume was $20 \mu \mathrm{L}$ and the photodiode array detector was set at $530 \mathrm{~nm}$ for anthocyanins [60]. Three biological replicates were analyzed for each sample type.

\section{Supplementary Information}

The online version contains supplementary material available at https://doi. org/10.1186/s12863-021-00959-2.

Additional file 1 Chrysanthemum flowers. (A) Capitulum. (B) Disk floret. (C) Ray floret.

Additional file 2. The DEGs specifically expressed in the mutant capitula Additional file 3. The DEGs specifically expressed in the normal capitula Additional file 4. The DEGs annotated with the "reproduction" GO term (GO:0000003)

Additional file 5. The DEGs enriched in the biological process GO category

Additional file 6. The DEGs enriched in the molecular function GO category

Additional file 7. The DEGs enriched in the cellular component GO category

Additional file 8. The enriched KEGG pathways among the DEGs downregulated in the mutant capitula

Additional file 9. The enriched KEGG pathways among the DEGs upregulated in the mutant capitula

Additional file 10. Important transcription factor genes substantially differentially expressed between the normal and mutant capitula

Additional file 11. Important photoperiod pathway genes in chrysanthemum

Additional file 12. The MADS-box genes identified in chrysanthemum

Additional file 13. The genes involved in the GA pathway in chrysanthemum

Additional file 14. Important anthocyanin biosynthesis pathway functional genes in chrysanthemum

Additional file 15 Primers used for the quantitative real-time PCR analysis of Chrysanthemum morifolium

\section{Abbreviations}

DEG: Differentially expressed gene; PCR: Polymerase chain reaction; GPCR: Quantitative PCR

\section{Acknowledgments}

We thank Robbie Lewis, MSc, from Liwen Bianji, Edanz Group China (www. liwenbianji.cn/ac), for editing a draft of this manuscript. 


\section{Availability of supporting data}

The Illumina reads have been deposited in the Sequence Read Archive (SRA) database at NCBI (http://www.ncbi.nlm.nih.gov/sra) and are available under study accession number SRP282697.

\section{Authors' contributions}

$H L, C L, D C$, and $Y W$ performed the research. $H L$ analyzed the data and prepared the manuscript. $\mathrm{CH}$ and $\mathrm{ML}$ guided the research. SG, XXC, JB, XH, and $X C$ provided assistance. All authors read and approved the final manuscript.

\section{Funding}

This research was supported by the Beijing Natural Science Foundation (6194033), the Natural Science Foundation of the Beijing Academy of Agriculture and Forestry Sciences (QNJJ201817), the Innovation Foundation of the Beijing Academy of Agriculture and Forestry Sciences (KJCX20200112), the National Natural Science Foundation of China (31901354). All the funding bodies didn't participate in the design of the study and collection, analysis, and interpretation of data and writing the manuscript.

\section{Availability of data and materials}

All data generated or analysed during this study are included in this published article and its supplementary information files.

\section{Ethics approval and consent to participate}

Not applicable.

\section{Consent for publication}

Not applicable.

\section{Competing interests}

The authors declare that they have no competing interests.

\section{Author details}

${ }^{1}$ Beijing Agro-Biotechnology Research Center, Beijing Academy of Agriculture and Forestry Sciences, Beijing Engineering Research Center of Functional Floriculture, Beijing, Key Laboratory of Agricultural Genetic Resources and Biotechnology, Beijing 100097, China. ${ }^{2}$ Beijing Vegetable Research Center, Beijing Academy of Agriculture and Forestry Sciences, Beijing 100097, China.

Received: 6 May 2020 Accepted: 5 January 2021

Published online: 25 January 2021

\section{References}

1. Su J, et al. Current achievements and future prospects in the genetic breeding of chrysanthemum: a review. Horticulture Research. 2019;6:109.

2. Ja, T.D.S., Chrysanthemum: advances in tissue culture, cryopreservation, postharvest technology, genetics and transgenic biotechnology. 2003. 21(8): p. 715-766.

3. Roberts WR, Roalson EH. Comparative transcriptome analyses of flower development in four species of Achimenes (Gesneriaceae). BMC Genomics. 2017;18(1):240

4. O'Maoileidigh DTEA. Gene network analysis of Arabidopsis thaliana flower development through dynamic gene perturbations. Plant J. 2015:83(2):34458.

5. Irish VF. The flowering of Arabidopsis flower development. Plant Journal for Cell \& Molecular Biology. 2010;61(6):1014-28.

6. Lyu J, et al. The floral Repressor GmFLC-like is involved in regulating flowering time mediated by low temperature in soybean. Int J Mol Sci. 2020

7. Smaczniak, C., et al., Developmental and evolutionary diversity of plant MADS-domain factors: Insights from recent studies. Development (Cambridge, England), 2012. 139: p. 3081-98.

8. Weiss D. Regulation of flower pigmentation and growth: multiple signaling pathways control anthocyanin synthesis in expanding petals. Physiol Plant. 2000;110(2):152-7

9. Elomaa, P., et al., Activation of Anthocyanin Biosynthesis in Gerbera hybrida (Asteraceae) Suggests Conserved Protein-Protein and Protein-Promoter Interactions between the Anciently Diverged Monocots and Eudicots. Plant Physiology. 133(4): p. 1831-1842.
10. Zhang $Y$, et al. Altered expression of CmNRRa changes flowering time of Chrysanthemum morifolium. Plant Biotechnol J. 2013;11(3):373-9.

11. Wei $Q$, et al. Control of chrysanthemum flowering through integration with an aging pathway. Nat Commun. 2017:8(1):829.

12. Cui-Hui, et al., The MADS transcription factor CmANR1 positively modulates root system development by directly regulating CmPIN2 in chrysanthemum. [J]. Horticulture research, 2018.

13. Yang, Y., et al., Interactions between WUSCHEL- and CYC2-like transcription factors in regulating the development of reproductive organs in Chrysanthemum morifolium, in international journal of molecular sciences. 2019.

14. Noda N, et al. Generation of blue chrysanthemums by anthocyanin B-ring hydroxylation and glucosylation and its coloration mechanism. Sci Adv. 2017:3(7):e1602785.

15. Akemi and Ohmiya, Molecular mechanisms underlying the diverse array of petal colors in chrysanthemum flowers. Breed Sci. 2018. 68(1):119-127.

16. Junyang, Y., et al., Transcriptome analysis of differentially expressed unigenes involved in flavonoid biosynthesis during flower development of Chrysanthemum morifolium 'Chuju'. Scientific Reports. 2018. 8(1): p. 13414-.

17. Shaokui, et al., Full-length transcriptome of Misgurnus anguillicaudatus provides insights into evolution of genus Misgurnus.[J]. Scientific reports, 2018.

18. Song $C$, et al. The Chrysanthemum nankingense genome provides insights into the evolution and diversification of Chrysanthemum flowers and medicinal traits. Mol Plant. 2018;11(12):1482-91.

19. Hirakawa $\mathrm{H}$, et al. De novo whole-genome assembly in Chrysanthemum seticuspe, a model species of chrysanthemums, and its application to genetic and gene discovery analysis. DNA Res. 2019;26:195-203.

20. Krizek BA, Meyerowitz EM. The Arabidopsis homeotic genes APETALA3 and PISTILLATA are sufficient to provide the B class organ identity function. Development. 1996:122(1):11-22.

21. Parcy F, Bomblies K, Weigel D. Interaction of LEAFY, AGAMOUS and TERMIN AL FLOWER1 in maintaining floral meristem identity in Arabidopsis. Development. 2002;129(10):2519-27.

22. Litt $A$, Kramer $E M$. The $A B C$ model and the diversification of floral organ identity. 2010. Semin Cell Dev Biol. 2010;21(1):129-37.

23. Wang $\mathrm{H}$, et al. Next-generation sequencing of the Chrysanthemum nankingense (Asteraceae) Transcriptome permits large-scale Unigene assembly and SSR marker discovery. PLoS One. 2013;8(4):e62293.

24. Ren, $L$., et al. A transcriptomic analysis of Chrysanthemum nankingense provides insights into the basis of low temperature tolerance. [J]. BMC Genomics, 2014, 15(1):844

25. Liu $H$, et al. Whole-transcriptome analysis of differentially expressed genes in the ray florets and disc florets of Chrysanthemum morifolium. BMC Genomics. 2016;17(1):398.

26. Liu $\mathrm{H}$, et al. Whole-Transcriptome analysis of differentially expressed genes in the vegetative buds, Floral Buds and Buds of Chrysanthemum morifolium. PLOS ONE. 2015;10:e0128009.

27. Marin-de LRN, et al. Integrating circadian and gibberellin signaling in Arabidopsis: possible links between the circadian clock and the AtGID1 transcription. Plant Signal Behav. 2011:6(9):1411-3.

28. Rodolfo Zentella ZZMP. Global analysis of DELLA direct targets in early gibberellin signaling in Arabidopsis. Plant Cell. 2007;19(10):3037-57.

29. Zhang $S$, et al. GASA5, a regulator of flowering time and stem growth in Arabidopsis thaliana. Plant Mol Biol. 2009:69(6):745-59.

30. Chen, S. and S. Wang, GLABRA2, A Common Regulator for Epidermal Cell Fate Determination and Anthocyanin Biosynthesis in Arabidopsis. Int J Mol Sci, 2019. 20(20)

31. Naing AH, Kim CK. Roles of R2R3-MYB transcription factors in transcriptional regulation of anthocyanin biosynthesis in horticultural plants. Plant Mol Biol. 2018;98(1):1-18

32. Li H, et al. MdMYB8 is associated with flavonol biosynthesis via the activation of the MdFLS promoter in the fruits of Malus crabapple. Hortic Res. 2020:7:19.

33. Weiss, D., et al., Stamens and Gibberellic Acid in the Regulation of Flavonoid Gene Expression in the Corolla of Petunia hybrida. Plant Physiology. 94(2): p. 511-515.

34. Qi T, et al. Arabidopsis DELLA and JAZ proteins bind the WD-repeat/bHLH/ MYB complex to modulate gibberellin and jasmonate signaling synergy. Plant Cell Plant Cell. 2014:26(3):1118-33.

35. Barendse, G.W.M., et al., GROWTH HORMONES IN POLLEN, STYLES AND OVARIES OF Petunia HYBRIDA AND OF LILIUM SPECIES. Acta Botanica Neerlandica, 1970. 19(2) 
36. Sasaki K, et al. Functional divergence within class B MADS-box genes TfGLO and TfDEF in Torenia fournieri Lind. Mol Genet Genomics. 2010;284(5):399414.

37. Lorenzo O, et al. ETHYLENE RESPONSE FACTOR1 integrates signals from ethylene and Jasmonate pathways in plant defense. Plant Cell. 2003;15(1): 165-78.

38. Qi T, et al. The Jasmonate-ZIM-domain proteins interact with the WDrepeat/bHLH/MYB complexes to regulate Jasmonate-mediated anthocyanin accumulation and trichome initiation in Arabidopsis thaliana. Plant Cell. 2011;23(5):1795-814.

39. Peng $Z$, et al. Brassinosteroid enhances jasmonate-induced anthocyanin accumulation in Arabidopsis seedlings. J Integr Plant Biol. 2011;53(8):632-40.

40. Alam I, et al. Comprehensive genomic survey, structural classification and expression analysis of $\mathrm{C} 2 \mathrm{H} 2$ zinc finger protein gene family in Brassica rapa L. PLoS One. 2019:14(5):e0216071.

41. Dathan and N., The Arabidopsis SUPERMAN protein is able to specifically bind DNA through its single Cys2-His2 zinc finger motif. Nucleic Acids Research. 2002. 30(22): p. 4945-4951.

42. Prunet $\mathrm{N}$, et al. SUPERMAN prevents class $\mathrm{B}$ gene expression and promotes stem cell termination in the fourth whorl of Arabidopsis thaliana flowers. Proc Natl Acad Sci U S A. 2017;114(27):201705977.

43. Yang $Y$, et al. The bZIP gene family in watermelon: genome-wide identification and expression analysis under cold stress and root-knot nematode infection. Peer J. 2019:7:e7878.

44. Gibalová A, et al. Characterization of pollen-expressed bzIP protein interactions and the role of ATbZIP18 in the male gametophyte. Plant Reproduction. 2017;30(1):1-17.

45. Wang $Y$, et al. The inhibitory effect of $A B A$ on floral transition is mediated by ABI5 in Arabidopsis. J Exp Bot. 2013;2:2.

46. Chen $C$, et al. MYB transcription repressors regulate plant secondary metabolism. Crit Rev Plant Sci. 2019;38(3):159-70.

47. Li J, An Y, Wang L. Transcriptomic analysis of Ficus carica peels with a focus on the key genes for anthocyanin biosynthesis, international journal of molecular sciences; 2020

48. Amírez, C.I.O., et al., Expression and function of the bHLH genes ALCATRAZ and SPATULA in selected Solanaceae species. Plant J, 2019. 99(4).

49. Schunter $C$, et al. Transcriptome analyses and differential gene expression in a non-model fish species with alternative mating tactics. BMC Genomics. 2014;15(1):167.

50. Conesa A, et al. Blast2GO: a universal tool for annotation, visualization and analysis in functional genomics research. Bioinformatics. 2005;21(18):3674-6.

51. Ye, J., WEGO: a web tool for plotting GO annotations. 2006. 34(Web Server issue): p. W293-W297.

52. Fourquin $\mathrm{C}$, et al. Evidence that CRABS CLAW and TOUSLED have conserved their roles in carpel development since the ancestor of the extant angiosperms. Proc Natl Acad Sci U S A. 2005;102(12):4649-54.

53. Samarskiĭ A. Claverie JM: the significance of digital gene expression profiles. Genome Res. 1997;7(10):986-95.

54. Endress PK. Angiosperm floral evolution: morphological developmental framework. Adv Bot Res. 2006:44:1-61.

55. Mortazavi A, et al. Mapping and quantifying mammalian transcriptomes by RNA-Seq. Nat Methods. 2008;5(7):621-8.

56. Li, J., et al., Long read reference genome-free reconstruction of a full-length transcriptome from Astragalus membranaceus reveals transcript variants involved in bioactive compound biosynthesis. 2017. 3: p. 17031.

57. Alamancos GP, et al. Leveraging transcript quantification for fast computation of alternative splicing profiles. RNA. 2015;21(9):1521-31.

58. Xue, C.M., et al., Reference gene selection for quantitative real-time PCR in Pandora neoaphidis. Journal of Agricultural Biotechnology, 2014.

59. Du, H., et al., Methylation mediated by an anthocyanin, O-methyltransferase, is involved in purple flower coloration in Paeonia. J Exp Bot, 2015. 66(21).

60. Revilla, E. And J.M. Ryan. Analysis of several phenolic compounds with potential antioxidant properties in grape extracts and wines by highperformance liquid chromatography-photodiode array detection without sample preparation 2000. 881(1): p. 461-469.

\section{Publisher's Note}

Springer Nature remains neutral with regard to jurisdictional claims in published maps and institutional affiliations.

\section{Ready to submit your research? Choose BMC and benefit from:}

- fast, convenient online submission

- thorough peer review by experienced researchers in your field

- rapid publication on acceptance

- support for research data, including large and complex data types

- gold Open Access which fosters wider collaboration and increased citations

- maximum visibility for your research: over $100 \mathrm{M}$ website views per year

At $\mathrm{BMC}$, research is always in progress.

Learn more biomedcentral.com/submissions 